\title{
De Morgan's laws and NEG-raising: a syntactic view
}

Research Article

\author{
Diego Gabriel Krivochen*
}

University of Reading, Reading, UK

Received 3 November, 2018; Accepted 28 January, 2019

\begin{abstract}
In this paper, we will motivate the application of specific rules of inference from the propositional calculus to natural language sentences. Specifically, we will analyse De Morgan's laws, which pertain to the interaction of two central topics in syntactic research: negation and coordination. We will argue that the applicability of De Morgan's laws to natural language structures can be derived from independently motivated operations of grammar and principles restricting the application of these operations. This has direct empirical consequences for the hypothesised relations between natural language and logic.
\end{abstract}

Keywords: negation • coordination $\bullet$ ATB movement $\bullet$ Coordinate Structure Constraint $\bullet$ De Morgan's laws

(c) Sciendo

\section{Introduction}

In logic, De Morgan's laws are rules of inference which determine equivalences between combinations of the negation [NEG henceforth] and the conjunction and disjunction operators [1, p. 25; 2]. We can illustrate them as follows:

1) a. $\sim(A \vee B) \leftrightarrow(\sim A \wedge \sim B)$

b. $\sim(A \wedge B) \leftrightarrow(\sim A \vee \sim B)$

It is not obvious that laws of inference like these, conceived within propositional logic and Boolean algebra, should work for natural languages. However, they seem to hold. When A and B are sentences (but not when we are dealing with quantified noun phrases [NPs] like no student; [3, p. 39; 4], which is exemplified in sentence (4)), De Morgan's laws are obeyed:

2) a. Mary doesn't write novels and compose lieder $\leftrightarrow b$. Mary doesn't write novels or Mary doesn't compose lieder 3) a. Mary doesn't write novels or compose lieder $\leftrightarrow b$. Mary doesn't write novels and Mary doesn't compose lieder 4) a. No student sang and danced $\neq$ b. No student sang or no student danced

At this point, the reader may legitimately wonder, as an anonymous reviewer did, whether 'we're only dealing with a morphological accident in English that makes the law hold to some degree'. This is not a trivial question, since it may undermine the validity of a syntactic approach. It is thus necessary to briefly look at the facts in a language that differs from English in all relevant respects. As is well known, Spanish is a pro-drop language, which means that it allows for null subjects in finite clauses. If we get overt subjects out of the equation, however, the facts remain equally robust:

$2^{\prime}$ ) a. No escribo novelas y compongo lieder $\leftrightarrow$ b. No escribo novelas ni compongo lieder NEG write $_{1 \text { Sgpres }}$ novels and compose ${ }_{1 \text { SgPres }}$ lieder $\leftrightarrow$ NEG write $_{1 \text { Sgpres }}$ novels conj-NEG compose ${ }_{1 \text { Sgpres }}$ lieder

\footnotetext{
*Corresponding author: Diego Gabriel Krivochen, E-mail: diegokrivochen@hotmail.com 
3') a. No escribo novelas o compongo lieder $\leftrightarrow$ b. No escribo novelas y no compongo lieder

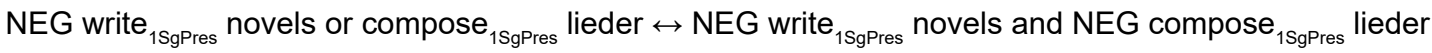

All we seem to have left is simply the interaction between NEG and clausal domains: the distribution of NEG over coordinated sentences [Ss] in conjunctive and disjunctive contexts. While the observation of the empirical facts is relatively straightforward, their explanation is far from that, given that (as pointed out above) the origin of De Morgan's laws is to be found in formal logic, not linguistics. In this context, it seems that two alternative routes are available to us if we aim to provide an account of sentences (2) and (3):

-We could follow Lakoff [5] and much subsequent work [6-8] in assuming that the structure of natural languages (their grammar, including an alphabet of symbols, rules, and conditions over rules) is isomorphic to the structure of the language of propositional logic. Or,

-We could try to derive the observation that natural language seems to follow these specific logical rules of inference from independently motivated conditions on linguistic structure.

There is a clear appeal to the first option, for both the formal semanticist and the syntactician. An isomorphism between natural language and formal logic would simplify syntactic theory and the syntax-semantics interface, in addition to making unambiguous rules of semantic interpretation directly correlate with rules of structure building and mapping in a perfectly monotonic compositional system. MacCartney and Manning [9] formulate the issue in very clear terms:

For a semanticist, the most obvious approach to NLI [natural language interpretation, D.G.K.] relies on full semantic interpretation: first, translate $p$ and $h$ into some formal meaning representation, such as first order logic (FOL), and then apply automated reasoning tools to determine inferential validity (p. 140)

But things are not always simple. MacCartney and Manning [9] point out that 'natural logic'-based computational systems failed to find proofs in an overwhelming $96 \%$ of the problems in the annual Recognizing Textual Entailment (RTE) test, an open-domain NLI task. Furthermore, they show that full semantic interpretation is not always a necessary condition to prove inferential validity, which may be well and good in computer-based NLP, but which certainly falls short when the issue is looked at from the viewpoint of the theory of natural language grammar with both descriptive and explanatory adequacy as goals. In this context, the appeal increases of independently motivated linguistic constraints on dependencies and their interaction as the reason why natural languages seem to be isomorphic to logical languages: formal properties of these two kinds of languages may converge in some specific conditions, but this does not mean that they are one and the same. In other words, the second route turns issues pertaining to the relation between natural and logical languages into an empirical question.

From a linguistic point of view, then, we can examine the conditions under which De Morgan's laws are obeyed in natural languages and even attempt an explanation of why they should hold for certain values of A and B (cf. sentence (1)). In order to do this, we need to examine two things: (a) the structure of coordination; and (b) the dynamics of NEG-raising.

\section{Conditions on coordination}

As for the structure of coordination, we need to consider how surface coordinations arise and what kinds of operations can apply to objects in coordinate structures. The relevant cases are all coordinated structures where the conjuncts are accessible to externally triggered operations (including extraction and agreement): in Krivochen and Schmerling [10] and Krivochen [11], we refer to this kind of coordinate structure as et-coordination (inspired by Latin coordination with et), and it includes both symmetric and asymmetric conjunction [12]. Et-coordination contrasts with que-coordination (whose name is inspired by Latin coordination with -que), in which the terms of the coordination are not accessible: the output of que-coordination is an atomic syntactic object. Et-coordination must be distinguished from que-coordination both syntactically and semantically. Whereas et-coordination of subjects requires plural verb agreement, as in sentence (5), que-coordination triggers singular agreement, as in sentence (6):

5) The sudden rise and the equally sudden fall of the stock market have economists worried. 
6) The sudden rise and equally sudden fall of the stock market has economists worried.

Compare also the Spanish examples in sentences (7) and (8):

7) La abrupta subida y la igualmente abrupta bajada de la the sudden rise and the equally sudden fall of the bolsa preocupan (ambas) al Gobierno. stock market worry (pl) (both) the Government 'The sudden rise and the equally sudden fall of the stock market (both) worry the Government.'

8) La abrupta subida e igualmente abrupta bajada de la bolsa the sudden rise and equally sudden fall of the stock-market preocupa al Gobierno. worries (sg) the Government

'The sudden rise and equally sudden fall of the stock market worries the Government.'

The English example (6) and the Spanish example (8) are both instances of que-coordination. In each of these examples, the conjoined NPs are understood as picking out a single entity: the 'fluctuation' of the stock market rather than individual rises and falls. Sentences (5) and (7), exhibiting et-coordination, are not syntactically or semantically equivalent to their que-coordinated counterparts: these sentences, in contrast to the que-coordinated examples in sentences (6) and (8), are not understood as referring to economists who are all concerned about the same stocks. The English and and the Spanish $y$ constitute a transparent domain, which is thus accessible to probing operations, including agreement and extraction. In sentences (6) and (8), in contrast, the que-coordination yields one entity semantically, which is not accessible to further operations-whence the singular verb agreement in those sentences. It is et-coordination that has a disjunctive counterpart, not que-coordination: et-coordination includes phenomena like Culicover's OM-sentences [13], in which an NP is et-coordinated with an S:

9) One more can of beer and l'm leaving

As Culicover notes [13, p. 18 et passim], OM-sentences can also have disjunctive versions:

10) A thousand cans of beer or I'm leaving. (Culicover's sentence (8))

The asymmetry in the conjuncts indicates et-coordination, insofar as both conjuncts must be accessible to a variety of inferences (which Culicover [13] refers to as conditional, consequential and incongruous; we will not get into details about these here as they are immaterial to the present argument). What matters here is that the availability of disjunction is restricted to et-coordination as a consequence of the accessibility of conjuncts: in order to have a choice between A or B, you need to have A and B as distinct entities, semantically and syntactically. In Krivochen and Schmerling [10], we proposed that candidates for disjunction have to meet both of the following requirements:

1. They must be distinct referentially, and

2. They must be distinct syntactic objects, in the sense that they are accessible separately for purposes of o syntactic operations

o semantic interpretation

Semantic interpretation includes relative ordering between events, as well as inferences such as consecutive or conditional readings (in Culicover's OM sentences, we have a disjunction of NP and $S$ in strictly categorial terms, but it is necessary to account for the fact that the first term of the coordination is interpreted eventively: give me NP or...S / give me NP and (after that)...S); it also includes the possibility of having a disjunctive variant. One test for etvs. que-coordination is thus the possibility of using or in place of and. Syntactic operations include verb agreement when the coordination appears as the subject of the sentence, and - crucially for our present purposes - reordering transformations, as with the possibility of having across-the-board (ATB) extraction from the conjuncts. This means, importantly, that the CSC applies to et-coordinated structures in both symmetric and asymmetric varieties [12]. 
Once we have restricted the object of our investigation to et-coordinated structures (i.e. true coordinations where the terms are distinct semantically and syntactically and the coordinated structure can be internally probed by operations triggered from the outside), we need to give some more details about the operations that yield the surface form of the coordination. Let us begin by looking at sentence (11), a pre-transformational string (a 'kernel sentence'):

11) John drinks beer and John drinks wine and John drinks gin

[14, p. 175] proposed a rule of conjunction reduction (CR), which - in slightly modified terms - applies across the board by identifying common objects in all conjuncts, root-adjoining the relevant object and deleting structurally low occurrences under identity. CR applied to sentence (11) yields sentence (12):

12) a. [S John drinks [[ ${ }_{S}$ and John drinks beer] [s and John drinks wine] $\left[_{S}\right.$ and John drinks gin]]]

b. John drinks beer, wine and gin

Note that if there is a transformational relation between the (a) and (b) cases in sentences (2) and (3), the surface structures arise only if there is some process of deletion under identity, which simplifies conjuncts by deleting repeated objects in parallel structural positions (in sentence (12), for instance, the subject John and the verb drink). As pointed out above, CR involves two steps: adjunction and deletion:

Conjunction Reduction [...] Chomsky-adjoins to the right or left of the coordinate node a copy of some constituent which occurs in all conjuncts, on a right or left branch, respectively, and then deletes the original nodes [14, p. 175].

Furthermore, Ross [14, p. 176] restricts CR in the following way:

It is important to note that Conjunction Reduction must work 'across the board'

A rule applies ATB if and only if it affects all terms of a coordinate structure (see also [15, p. 32] for a generalised formalisation of the ATB condition); in this case, the elements affected by CR must occur in each conjunct. It should be apparent that ATB rules can apply if and only if conjuncts can be internally affected by syntactic operations; in other words, only et-coordinated structures can be affected by ATB rules.

Coordinate structures are subject to locality conditions, such that the extraction of objects from conjuncts is rather heavily restricted. The relevant condition is the Coordinate Structure Constraint (CSC; [14]), which filters out non-ATB extraction from coordinate structures. Postal [16, p. 83] elaborates on Ross's original formulation in a way that is relevant to our present discussion:

It seems correct to divide Ross's original formulation of the CSC into separate principles. The one I called the Conjunct Constraint [...] forbids the extraction of coordinate conjuncts themselves. The other, the CSC, bans (non-

ATB) extraction from true conjuncts.

The classical examples of ATB extraction from coordinate structures involve leftwards movement (wh-movement) or rightwards movement (right node raising, RNR henceforth), which we exemplify in sentences (13) and (14), respectively:

13) What ${ }_{i}$ did Mary buy $t_{i}$ and John break $t_{i}$ ?

14) Mary bought $t_{i}$ and John broke $t_{i}$ a lovely China vasei

The ATB condition on extraction determines that the extracted object corresponds to the gaps in both conjuncts, which we have indicated via indexed traces. If extraction from a coordinate structure does not proceed in an ATB manner (i.e. targeting objects in both conjuncts), the result is ungrammatical:

13') *What did Mary buy a new TV and John break $t_{i}$ ?

14') *Mary bought $t_{i}$ and John broke a new TV a lovely China vase ${ }_{i}$ 
The requirement that extraction from et-coordinated structures be ATB will play a crucial role in our treatment of negation, and our attempt to deduce the applicability of De Morgan's laws to natural language from independently motivated conditions in the grammar. We will provide arguments in favour of the hypothesis that De Morgan's laws in natural language are instances of syntactic NEG raising out of coordinate structures.

\section{NEG-raising}

NOT-transportation [17], better known today as NEG-raising (as per $[18,19])$ was originally formulated as a cyclic syntactic transformational rule that yielded raising of a negative operator in multi-clausal structures while maintaining the low-NEG interpretation:

Transposition of NOT(EVER) to Main Verb (Partly obligatory)

Under certain conditions (e.g., after verbs like WANT or THINK which are themselves not negated), a NOT in the embedded sentence may be moved in front of the main verb.

Relevant examples are like sentences (15) and (16) (from Fillmore [17, p. 220])

15) a. I think that he will not come $\rightarrow$ NEG-raising

b. I don't think that he will not come

16) a. I want him not to come $\rightarrow$ NEG-raising

b. I don't want him not to come

The cyclic nature of NEG-raising allows for monotonic embedding, such that in sentence (17),

17) I don't believe that he wants me to think that he did it

not is 'originally associated to did it' [17, p. 220, fn. 12].

Collins and Postal $[20,21]$ have reinvigorated the syntactic treatment of NEG-raising, providing novel arguments to distinguish between at least three cases:

a) Standard cases of NEG-raising: cyclic predicate-to-predicate raising

b) Negative quantifier cases: NEG raised to a predicate interacting with an embedded quantified NP

c) Cloud of the unknown cases [22].

Here, we will deal only with standard cases, examples in which NEG is interpreted as having scope over a verbal predicate (a NEG-V reading, in Carden's [23] terms). We will further restrict our inquiry to two variants of the coordinated structure matrix and embedded (as in examples (18a) and (18b), respectively):

18) a. Mary doesn't write novels and compose lieder $(=(2 a))$

b. I don't think that Mary writes novels and composes lieder

The abstract structures corresponding to these examples are presented in (19a) and (19b):

19) a. [...NEG...[V...and...V]]

b. [...NEG V...[V...and...V]]

A salient feature of these bracketed analyses is that NEG is outside the coordinate structure itself. This is the key aspect of our proposal: the syntax of De Morgan-complying negative coordinate structures (De Morgan coordinations, henceforth) is parallel to that of leftwards ATB extraction (as in the case of wh-movement or RNR out 
of coordinate structures; see sentences (13)-(14)). This is possible if, following previous authors [17, 18, 20, 21], NEG-raising in the standard cases is formalised as a syntactic reordering rule (in the sense of Ross [14, p. 427]); in this case, NEG-raising must be subjected to the same requirements (filters, conditions, and constraints) as other rules of the grammar. This means, crucially, that NEG raising out of coordinated structures is subject to the CSC and, therefore, is only grammatical if it applies ATB.

Extraction from parallel coordinated structures [24] targets a position outside the terms of the coordination; this is true of both leftwards and rightwards extraction. Thus, sentences (13) and (14) above involve the reordering of an NP which is interpreted in positions internal to both terms of the coordination to a peripheral position outside the domain of the coordination. Schmerling [12] provides ample evidence for a distinction between symmetric and asymmetric coordination, which can be exemplified in sentences (20) and (21), respectively (taken from Schmerling [12, p. 211]):

20) a. Paris is the capital of France and Rome is the capital of Italy = b. Rome is the capital of Italy and Paris is the capital of France

21) a. Harry stood up and objected to the proposal $\neq$ b. Harry objected to the proposal and stood up

Let us assume, in the spirit of 'mixed computation' advocated for previously [10,11], that asymmetric coordination of $\mathrm{X}$ and $\mathrm{Y}$ receives a hypotactic phrase structural analysis [X [and [Y]]], whereas symmetric coordination is paratactic - computationally finite state - and branches $n$-arily (in the case of two coordinands, $[[X]$ [and] [Y]]); recall that both are instances of et-coordination. Because there is a temporal ordering implicature associated to the terms in examples (13) and (14) (such that John breaks the vase after Mary has bought it; just like in example (20a), Harry stands up and then objects to the proposal), we will assume we are in the presence of asymmetric coordination. Then, we can diagram the structures corresponding to examples (13) and (14) above as follows:

22)

a)

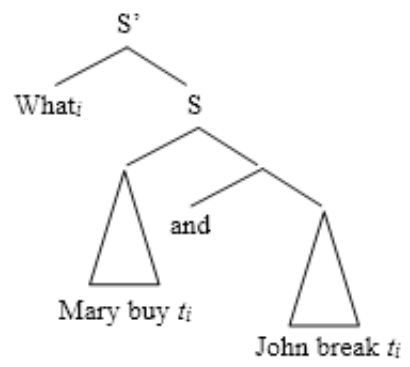

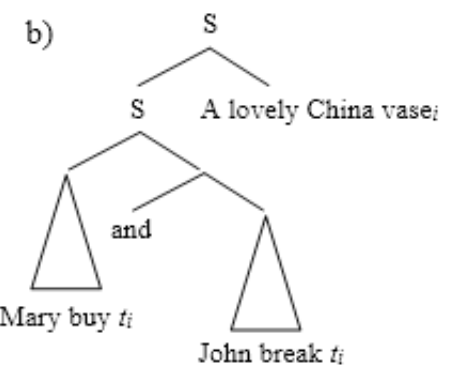

In example (22a), the S structure is extended to $\mathrm{S}^{\prime}$ (or Tense Phrase TP to Complementiser Phrase CP, or S to COMP[lementiser]... those distinctions are inconsequential to our point), whereas in example (22b), the Right Noderaised NP is Chomsky-adjoined to S. In both cases, however, it is necessary that the reordered element (whatever its category) has an occurrence in both conjuncts: in that way, the rule (wh-movement and RNR, respectively) can apply in an ATB manner and the output structure will not violate the CSC. We argue here that NEG moves out of coordinated structures in exactly the same way.

Let us first consider the case where a coordinate structure appears as the complement to a verb that governs NEG-raising. If NEG-raising is a syntactic reordering transformation, then in coordinate structures, it must apply ATB: this means that the rule cannot just target a single term of the coordination, but also that the reordered element will receive an interpretation in both conjuncts (in examples (13) and (14), the reordered NP is interpreted as the Object of buy and break). The derivation for the structural description corresponding to the interpretation of $I$ don't think Mary writes novels and/or composes lieder (which, incidentally, is a symmetric coordination) goes along the following lines:

23) a. $\left[_{S}\right.$ I think [ $[$ [Mary write novels] and [Mary compose lieder]]] (Deep Structure)

b. [ I think [ $[$ [Mary NEG write novels] and [Mary NEG compose lieder]]] (NEG-placement)

c. [ $\left[_{S}\right.$ I think [S Mary [S [Mary NEG write novels] and [Aary NEG compose lieder]]]] (Conjunction Reduction)

d. [S I NEG think [S Mary [S [Aary $t_{N E G}$ write novels] and [Aary $t_{N E G}$ compose lieder]]] (ATB NEG-raising)

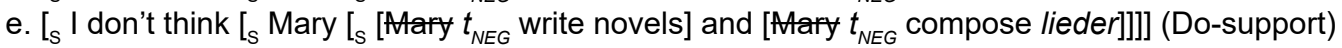


Step (23d) is what we are most interested in. After CR has applied ATB, Chomsky-adjoining [Mary], NEG-raising applies, again in an ATB fashion, reordering NEG in both conjuncts into the matrix clause. Note that CR cannot apply to the segment [Mary NEG] because it is not a constituent, and CR, as defined by Ross [14] applies to shared constituents in coordinate structures. In example (23d), the crucial step, we have NEG being reordered out of both conjuncts by NEG-raising, to the matrix predicate [think]. Because NEG cannot receive a morphological exponent in configuration (23d), do-support is inserted as a last resort to yield a legitimate representation.

If, in a polystratal model with pre- and post-transformational structures, the semantic interpretation of a sentence depends on both ([25]; see Schmerling [26]: xii-xiii for critical discussion), the surface form of the sentence I don't think Mary writes novels or compose lieder contains information necessary for the reconstruction of the position of NEG in the embedded coordinated clauses ${ }^{1}$; this is the same procedure that is required to interpret a lovely China vase as the Direct Object of both verbs buy and break in (14).

It is crucial to note that the derivation we have proposed for NEG-raising (which classically proceeds in a cyclic fashion from predicate to predicate) can be generalised to examples where there is no matrix predicate, yet negation outside a coordinate structure is interpreted distributively, as stated in De Morgan's laws. The crucial point here is that we can derive this reading from independently motivated conditions of grammar. Concretely, we refer to examples like sentence (24):

24) John doesn't work or study

The derivation we propose for sentence (24) goes along the lines of example (25):

25) a. [S [John work] and [John study]] (Deep Structure)

b. [S [John NEG work] and [John NEG study]] (NEG-placement)

c. [s John [s [John NEG work] and [John NEG study]] (Conjunction Reduction)

d. [S John NEG [ [John $t_{N E G}$ work] and [John $t_{N E G}$ study]] (ATB NEG-raising)

e. [S John doesn't [ ${ }_{S}$ [ $t_{N E G}$ work] and [Jo $t_{N E G}$ study] (Do-support)

If the requirement that operations on coordinate structures be ATB (as stated by the CSC) is a rule of the grammar of English (and Spanish, presumably), we can account for the fact that De Morgan's laws hold in specific instances of negation over coordinated structures in terms of independently motivated syntactic constraints and operations.

\section{Conclusions and further prospects}

Let us summarise our proposal. De Morgan's laws applied to negation in natural languages as a logical operator can be construed as NEG-raising, if NR is a syntactic operation [20] and thus must obey Ross's [14] CSC. This means that if we have a structural description of the kind $(\sim p \wedge \sim q)$, we can NEG-raise ATB outside the coordinate structure (given that NEG-raising is cyclic; see [17, p. 220] for the first explicit claim that Not-transportation operates from embedded clause to matrix clause; also [19, p. 130] and get $\sim(p \wedge q)$ if $p$ and $q$ are $S$ nodes. The same holds for the disjunctive coordinator $\mathrm{v}$, since it is structurally parallel to $\wedge$ in et-coordination.

This approach makes some concrete empirical predictions, among which we can count the following:

a) In $\sim(p \wedge q)$, NEG must necessarily be interpreted as distributing between the conjuncts in et-coordination, because NEG-raising must have applied ATB

1 Collins and Postal's ([20; 21, pp. 5-6]) analysis requires what they refer to as an interpretation property of NEG:

An NEG is interpreted only in its position of origin

The descriptive adequacy of this statement is exactly the same as the proposal that semantic interpretation takes place before the application of any reordering transformations. While it seems to be correct that 'raising of NEG out of its position of origin in A to some higher position has no effect on the semantics of $A$ or of any other constituent' $[21, p .6]$, an adequate theory of NEG-raising needs to be able to account for the pragmatic effects that differ in NEG-raised clauses and NEG-in situ ([19; 22; 32], among many others). 'I don't think that you'll come' versus 'I think that you won't come' may satisfy the same model [20, Ch. 3], but they also imply different degrees of commitment on the part of the subject with respect to the propositional content of the embedded clause. 
That is, it is not possible to interpret sentence (26a) as either sentence (26b) or sentence (26c):

26) a. John doesn't believe/suppose/imagine (that) Mary will have wine and champagne during dinner

b. John believes/supposes/imagines (that) Mary won't have wine and Mary will have champagne during dinner

c. John believes/supposes/imagines (that) Mary will have wine and Mary won't have champagne during dinner

Note that the interpretation in sentence (26b) has NEG only in the first conjunct, whereas sentence (26c) has NEG only in the second conjunct. Neither is an adequate paraphrase of the interpretation of sentence (26a); in the approach proposed here, this is because if NEG-raising applies to a coordinate structure, it must apply in an ATB manner (i.e. to all terms of the coordinate structure).

b) In and not structures [26, p. 87], NEG cannot raise, because it would violate the CSC

Here, we agree with Schmerling's [26] proposal that and not is a basic expression of the language, as opposed to considering it a derived expression, the result of concatenating and and not. Schmerling points out that

And not in fact has a quite general distribution, serving to coordinate expressions of a number of non-clausal categories that do not otherwise contain expressions with initial not

Some examples proposed by Schmerling [26, pp. 87-88] to illustrate her point are the following:

27) a. I saw John and not Mary.

b. *I saw not Mary.

28) a. The leaves turned yellow and not orange.

b. *The leaves turned not orange.

29) a. I put the book on the table and not on the shelf.

b. *I put the book not on the shelf.

In this context, consider the sentences in (30):

30) a. John wants beer and not gin

b. *John doesn't want beer and gin (bad on a NEG-raising reading)

c. *John doesn't want beer or gin (idem ant.)

It is not possible to relate sentence (30a) to sentence (30b) or (30c) by means of NEG-raising and maintain the semantic properties related to and not. The impossibility of manipulating NEG as an independent terminal for purposes of reordering transformations, including NEG-raising, would follow directly if NEG is not a terminal to begin with in and not; NEG-raising is not formulated for conjunctive elements (like and not); rather, it reorders a polarity operator (just not).

c) Furthermore, if the counter-arguments proposed by Postal [16] and Schmerling [27] against Goldsmith [28], Lakoff [29] and $\mathrm{Na}$ and Huck [30] are correct, then we expect example (27) to be grammatical in an NEG-raising reading (see also Schmerling [26, p. 88]):

31) a. John wants not beer, but he wants gin

b. John doesn't want beer, but he wants gin

In sentence (31), not is not part of a basic expression but not; we would expect it to be possible to reorder NEG independently given a syntactic approach to NEG-raising. These cases are structurally parallel to Goldsmithsentences (which feature the conjunction and not): 
32) How many lakes can we destroy and not arouse public antipathy?

In sentence (32), the CSC is irrelevant because reordering takes place within the first conjunct only: there is no reordering of [how many lakes] to a structural position that dominates both conjuncts; reordering does not involve at all the second term of the coordination.

The goal of this paper was to motivate the application of a law of the propositional calculus to natural language structure. It is important to remember that, unless an isomorphism is assumed between natural language and firstorder logic, there is no a priori reason why the properties of the propositional logic or a higher-order logical structure should hold for natural language (see Partee [31] for extensive discussion). In this paper, we attempted to take one property of the interaction between logical connectives and negation and motivate its applicability to natural language, appealing to independently motivated rules of the grammar. The relation between natural language and logic thus becomes an empirical problem rather than a philosophical one, a consequence that we deem highly desirable.

\section{References}

[1] Barwise, J., 1977. An introduction to first-order logic. In Barwise, J. (Ed.), Handbook of mathematical logic. London: Elsevier, pp. 5-46.

[2] McCawley, J. D., 1993. Everything that linguists have always wanted to know about logic...but were ashamed to ask. $2^{\text {nd }}$ edn. London: Blackwell.

[3] Collins, C., Postal, P., 2016. Interclausal NEG raising and the scope of negation. <https://ling.auf.net/ lingbuzz/002891>.

[4] Zwarts, F., 1996. Facets of negation. In Van der Does, J., van Eijck, J. (Eds.), Quantifiers, logic, and language. Stanford, California: CSLI, pp. 385-421.

[5] Lakoff, G., 1970. Linguistics and natural logic. Synthese, 22(1-2), 151-271.

[6] van Benthem, J., 1988. The semantics of variety in categorial grammars. In Buszkowski, W., Marciszewski, W., van Benthem, J. (Eds.), Categorial grammar. Amsterdam: John Benjamins, pp. 33-55.

[7] van Benthem, J., 1991. Language in action: categories, lambdas and dynamic logic. Studies in Logic. North-Holland.

[8] Sánchez Valencia, V., 1991. Studies on natural logic and categorial grammar. PhD thesis, University Amsterdam.

[9] MacCartney, B., Manning, C., 2009. An extended model of natural logic. In Proceedings of the eighth international conference on computational semantics, 140-156. Tilburg, The Netherlands: Association for Computational Linguistics. <http:// www.aclweb.org/anthology/W09-3714>

[10] Krivochen, D., Schmerling, S. F., 2016. Two kinds of coordination and their theoretical implications: rethinking structural uniformity. Ms. <https://www. academia.edu/13207764/_2016_Two_kinds_of_ coordination_and_their_theoretical_implications>

[11] Krivochen, D., 2016. Divide and...conquer? On the limits of algorithmic approaches to syntactic- semantic structure. Czech and Slovak Linguistic Review, 1, 15-37.

[12] Schmerling, S. F., 1975. Asymmetric conjunction and rules of conversation. In Cole, P., Morgan, J. (Eds.), Syntax and semantics, Vol. 3: Speech Acts, 211-231. New York: Academic Press.

[13] Culicover, P. W., 2013. OM-sentences. In Culicover, P. W., Explaining syntax, 15-52. Oxford: OUP.

[14] Ross, J. R., 1967. Constraints on variables in Syntax. PhD dissertation, MIT.

[15] Williams, E., 1978. Across-the-Board rule application. Linguistic Inquiry, 9(1), 31-43.

[16] Postal, P. M., 1998. Three investigations on extraction. Cambridge, Mass: MIT Press.

[17] Fillmore, C., 1963. The position of embedding transformations in a grammar. Word, 19(2), 208231.

[18] Horn, L. R., 1972. On the semantic properties of logical operators in English. PhD Dissertation, University of California at Los Angeles.

[19] Horn, L. R., 1978. Remarks on Neg-raising. In Cole, P. (Ed.), Syntax and semantics 9: pragmatics, 129220. New York: Academic Press.

[20] Collins, C., Postal, P., 2014. Classical NEG raising. Cambridge, Mass: MIT Press.

[21] Collins, C., Postal, P. 2017. Interclausal NEG raising and the scope of negation. Glossa: Journal of General Linguistics, 2(1), 1-29.

[22] Horn, L. R., 2014. The cloud of unknowing. In Hoeksema, J., Gilbers, D. (Eds.), Black book: a festschrift in honor of Frans Zwarts. Groningen: University of Groningen, pp. 78-96.

[23] Carden, G., 1970. A note on conflicting idiolects. Linguistic Inquiry, 1(3), 281-290.

[24] Goodall, G., 1984. Parallel structures in syntax. PhD Thesis, University of California, San Diego.

[25] Chomsky, N., 1970. Deep structure, surface structure, and semantic interpretation. In Jakobson, 
R., Kawamoto, S. (Eds.), Studies in general and oriental linguistics presented to Shirô Hattori on the occasion of his sixtieth birthday. Tokyo: TEC Corporation for Language and Education Research, pp. 52-91.

[26] Schmerling, S. F., 2018. Sound and grammar: towards a Neo-Sapirian theory of language. London: Brill.

[27] Schmerling, S. F., 1972. Apparent counterexamples to the coordinate structure constraint: a canonical conspiracy. Studies in the Linguistic Sciences, 2(1), 91-104.

[28] Goldsmith, J., 1985. A principled exception to the coordinate structure constraint. Papers from the general session at the twenty-first regional meeting of the Chicago Linguistic Society. University of Chicago, Illinois. Available online at <https://www. researchgate.net/publication/244998803_A_ Principled_Exception_to_the_Coordinate_
Structure_Constraint> [Accessed on 13/6/2015].

[29] Lakoff, G., 1986. Frame semantic control of the coordinate structure constraint. Proceedings of the 21st Annual Meeting of the Chicago Linguistic Society.

[30] Na, Y., Huck, G., 1992. On extracting from asymmetrical structures. In Brentari, D., Larson, G. N., MacLeod, L. A. (Eds.), The joy of grammar: a festschrift in honor of James D. McCawley. Amsterdam: John Benjamins, pp. 251-274.

[31] Partee, B., 2011. Formal semantics: origins, issues, early impact. In Partee, B., Glanzberg, M., Šḳilters, J. (Eds.), Formal semantics and pragmatics. Discourse, context, and models. The Baltic yearbook of cognition, logic, and communication, vol. 6. Manhattan: New Prairie Press, pp. 1-52.

[32] Zeijlstra, H., 2018. Does Neg-raising involve Negraising? Topoi, 37(3), 417-433. 